\title{
QUASI-ANTICHAIN CHERMAK-DELGADO LATTICES OF FINITE GROUPS
}

\author{
BEN BREWSTER, PETER HAUCK, AND ELIZABETH WILCOX
}

Dedicated to Otto H. Kegel for the occasion of his eightieth birthday.

\begin{abstract}
The Chermak-Delgado lattice of a finite group is a dual, modular sublattice of the subgroup lattice of the group. This paper considers groups with a quasi-antichain interval in the Chermak-Delgado lattice, ultimately proving that if there is a quasi-antichain interval between subgroups $L$ and $H$ with $L \leq H$ then there exists a prime $p$ such that $H / L$ is an elementary abelian $p$-group and the number of atoms in the quasi-antichain is one more than a power of $p$. In the case where the Chermak-Delgado lattice of the entire group is a quasi-antichain, the relationship between the number of abelian atoms and the prime $p$ is examined; additionally, several examples of groups with a quasi-antichain Chermak-Delgado lattice are constructed.
\end{abstract}

This paper pursues the nature of the Chermak-Delgado lattice of a finite group. The Chermak-Delgado lattice was introduced by Chermak and Delgado [4. Isaacs [6] re-introduced the lattice, sparking further study that resulted in [3] and [2]. In this article, we provide three primary contributions to the study of ChermakDelgado lattices: a description of the structure of groups with a quasi-antichain (defined below) as an interval in the Chermak-Delgado lattice, results that narrow the possible structure of a quasi-antichain realized as a Chermak-Delgado lattice, and examples to illustrate the breadth of possibilities. Among these contributions is a proof that if a Chermak-Delgado lattice has an interval which is a quasi-antichain then the width must be a power of a prime plus 1 .

Throughout the article, let $G$ be a finite group and $p$ be a prime. The ChermakDelgado lattice of a finite group $G$ consists of subgroups $H \leq G$ such that $\mid H \| C_{G}(H)$ is maximal among all subgroups of $G$. For any subgroup $H$ of $G$, the product $|H|\left|C_{G}(H)\right|$ is called the Chermak-Delgado measure of $H$ (in $G$ ) and is denoted by $m_{G}(H)$; if the group $G$ is clear from context then we write simply $m(H)$. To denote the maximum possible Chermak-Delgado measure of a subgroup in $G$ we write $m^{*}(G)$ and we refer to the set of all subgroups with measure attaining that maximum as the Chermak-Delgado lattice of $G$, or $\mathcal{C D}(G)$.

It is known that the Chermak-Delgado lattice is a modular self-dual lattice and if $H, K \in \mathcal{C D}(G)$ then $H K=K H=\langle H, K\rangle$. The duality of the Chermak-Delgado lattice is a result of the fact that if $H \in \mathcal{C D}(G)$ then $C_{G}(H) \in \mathcal{C D}(G)$ and $H=$ $C_{G}\left(C_{G}(H)\right)$. Moreover, if $M$ is the maximum subgroup in the Chermak-Delgado lattice of a group $G$ then the Chermak-Delgado lattices of $G$ and $M$ coincide. It is additionally known that the co-atoms in the Chermak-Delgado lattice are normal in $M$ and consequently the atoms, as centralizers of normal subgroups, are also normal in $M$. In [2], groups whose Chermak-Delgado lattice is a chain were studied; a chain of length $n$, where $n$ is a positive integer, is a totally ordered lattice with 
$n+1$ subgroups. We call a lattice consisting of a maximum, a minimum, and the atoms of the lattice a quasi-antichain and the width of the quasi-antichain is the number of atoms. A quasi-antichain of width 1 is also a chain of length 2 .

Let $L \leq H \leq G$; we use $\llbracket L, H \rrbracket$ to denote the interval from $L$ to $H$ in a sublattice of the lattice of subgroups of $G$. If $\llbracket L, H \rrbracket$ is an interval in $\mathcal{C D}(G)$ then the duality of the Chermak-Delgado lattice tells us that $\llbracket C_{G}(H), C_{G}(L) \rrbracket$ is an interval in $\mathcal{C D}(G)$. Of course, these intervals may overlap or even coincide exactly. In Section 1 we make no assumption about the intersection of $\llbracket L, H \rrbracket$ and $\llbracket C_{G}(H), C_{G}(L) \rrbracket$; in the final two sections we study the situation where these two intervals not only are equal, but are the entirety of $\mathcal{C D}(G)$.

\section{Quasi-antichain Intervals in Chermak-Delgado Lattices}

Let $G$ be a group with $L<H \leq G$ such that $\llbracket L, H \rrbracket$ is an interval in $\mathcal{C D}(G)$. The main theorem of this section establishes that if $\llbracket L, H \rrbracket$ is a quasi-antichain of width $w \geq 3$ then there exists a prime $p$ and positive integers $a, b$ with $b \leq a$ such that $H / L$ is an elementary abelian $p$-group with order $p^{2 a}$ and $w=p^{b}+1$. To make the role of the duality more transparent in the proofs, set $H^{*}=C_{G}(L)$ and $L^{*}=C_{G}(H)$. Observe that $C_{G}\left(H^{*}\right)=L$ and $C_{G}\left(L^{*}\right)=H$.

We start with a general statement about subgroups that are in the interval $\llbracket L, H \rrbracket$ in $\mathcal{C D}(G)$. Let $p$ be a prime dividing the index of $L$ in $H$. For this result, we remind the reader that the notation $\Omega_{k}(M)$, where $k$ is a positive integer and $M$ is any group, denotes the subgroup of $M$ generated by the elements whose order divides $p^{k}$.

The hypothesis of Proposition 1 may initially sound restrictive: We require that $G$ be a group with $\llbracket L, H \rrbracket$ in $\mathcal{C D}(G)$ such that $\left[H H^{*}, H H^{*}\right] \leq L \cap L^{*}$. Note that $L \unlhd H$ and $L^{*} \unlhd H^{*}$, so the quotient groups described in Proposition 1 are welldefined. Moreover, notice that the hypotheses of the proposition occur when $G$ is a $p$-group of nilpotence class 2 and $H=G \in \mathcal{C D}(G)$.

Proposition 1. Let $G$ be a group with an interval $\llbracket L, H \rrbracket$ in $\mathcal{C D}(G)$ such that $\left[H H^{*}, H H^{*}\right] \leq L \cap L^{*}$. Suppose that $p$ is a prime dividing $|H / L|$. The subgroups $A_{k}(H), B_{k}(H) \leq H$ where $A_{k}(H) / L=\Omega_{k}(H / L)$ and $B_{k}(H)=\left\langle x^{p^{k}} \mid x \in H\right\rangle L$ are members of $\mathcal{C D}(G)$ for all positive values of $k$, as are the similarly defined subgroups $A_{k}\left(H^{*}\right), B_{k}\left(H^{*}\right)$ of $H^{*}$.

Proof. Let $k$ be a positive integer. Without loss of generality, assume that $\left|A_{k}(H) / L\right| \geq$ $\left|A_{k}\left(H^{*}\right) / L^{*}\right|$. We first show that $C_{G}\left(A_{k}(H)\right)=B_{k}\left(H^{*}\right)$ and that $A_{k}(H) \in \mathcal{C D}(G)$.

Observe that if $x \in H$ and $y \in H^{*}$ then $[x, y] \in\left[H, H^{*}\right] \leq L \cap L^{*}=C_{G}\left(H^{*}\right) \cap$ $C_{G}(H)$. Therefore $\left[x^{p}, y\right]=\left[x, y^{p}\right]$ whenever $x \in H$ and $y \in H^{*}$. Moreover, if $x \in A_{k}(H)$ then $x^{p^{k}} \in L$, so $\left[x^{p^{k}}, y\right]=1$ for all $y \in C_{G}(L)=H^{*}$. Thus if $x \in A_{k}(H)$ and $y$ is a generator of $B_{k}\left(H^{*}\right)$ then $[x, y]=1$, therefore $B_{k}\left(H^{*}\right) \leq C_{G}\left(A_{k}(H)\right)$.

Since the quotient $H / L$ is abelian, $\left|A_{k}(H) / L\right|=\left|H / B_{k}(H)\right|$ or equivalently $\left|A_{k}(H) / L\right|\left|B_{k}(H)\right|=|H|$. The same is true regarding $\left|H^{*}\right|$ and the subgroups $A_{k}\left(H^{*}\right), B_{k}\left(H^{*}\right)$. Thus the measure of $A_{k}(H)$ in $G$ can be calculated as follows:

$$
\begin{aligned}
m\left(A_{k}(H)\right)=\left|A_{k}(H)\right|\left|C_{G}\left(A_{k}(H)\right)\right| & \geq\left|A_{k}(H)\right|\left|B_{k}\left(H^{*}\right)\right| \\
& =\left|A_{k}(H) / L\right||L|\left|B_{k}\left(H^{*}\right)\right| \\
& \geq\left|A_{k}\left(H^{*}\right) / L^{*}\right||L|\left|B_{k}\left(H^{*}\right)\right| \\
& =\left|H^{*}\right||L| \\
& =\left|C_{G}(L)\right||L|=m^{*}(G) .
\end{aligned}
$$


Therefore each inequality above is actually an equality, with $C_{G}\left(A_{k}(H)\right)=B_{k}\left(H^{*}\right)$ and $\left|A_{k}(H) / L\right|=\left|A_{k}\left(H^{*}\right) / L^{*}\right|$. Additionally $A_{k}(H), B_{k}\left(H^{*}\right), A_{k}\left(H^{*}\right), B_{k}(H) \in$ $\mathcal{C D}(G)$.

For the rest of the paper, we study intervals that are quasi-antichains. Ultimately we will use Proposition 1 to show that $A_{1}=H$ and $A_{1}^{*}=H^{*}$ in the case that $\llbracket L, H \rrbracket$ is a quasi-antichain of width $w \geq 3$. The next two propositions establish important facts about the atoms of a quasi-antichain interval in $\mathcal{C D}(G)$, as well as show that such an interval satisfies the hypothesis of Proposition 1.

Let $\llbracket L, H \rrbracket$ be a quasi-antichain of width $w$ throughout the remainder of the article. Let the $w$ atoms of the quasi-antichain be denoted by $K_{1}, K_{2}, \ldots, K_{w}$. The interval $\llbracket L^{*}, H^{*} \rrbracket$ is also a quasi-antichain in $\mathcal{C D}(G)$, with atoms $C_{G}\left(K_{i}\right)$ where $1 \leq i \leq w$. For each $i$, let $K_{i}^{*}=C_{G}\left(K_{i}\right)$ so that $C_{G}\left(K_{i}^{*}\right)=K_{i}$.

Proposition 2. If $K_{1}, K_{2}$ are distinct atoms of the quasi-antichain then $K_{i} \unlhd H$ for $i=1,2, L \unlhd H$, and $\left[K_{1}, K_{2}\right] \leq L$ and analogously for $H^{*}, K_{1}^{*}, K_{2}^{*}$, and $L^{*}$. Moreover, $\left|K_{1}: L\right|=\left|K_{2}^{*}: L^{*}\right|$.

If $w \geq 3$ then $K_{i} / L \cong K_{j} / L$ and $K_{i}^{*} / L^{*} \cong K_{j}^{*} / L^{*}$ for all $i, j$ with $1 \leq i, j \leq w$. Furthermore:

$$
|H / L|=\left|H / K_{1}\right|^{2}=\left|H^{*} / K_{1}^{*}\right|^{2}=\left|H^{*} / L^{*}\right| .
$$

Proof. Let $K_{1}, K_{2} \in \mathcal{C D}(G)$ with $L<K_{i}<H$ for $i=1,2$. Because the interval $\llbracket L, H \rrbracket$ is a quasi-antichain, $H=K_{1} K_{2}$ and $K_{1} \cap K_{2}=L$. From this structure and because $H$ cannot equal $K_{1} K_{1}^{h}$ for $h \in H$, it follows that $K_{1} \unlhd H$ (similarly for $\left.K_{2}\right)$. Therefore $L \unlhd H$ and $\left[K_{1}, K_{2}\right] \leq L$. The equality $m^{*}(G)=m(H)=m\left(K_{2}\right)$ implies

$$
\frac{\left|K_{2}\right|\left|K_{1}\right|}{|L|}\left|C_{G}(H)\right|=|H|\left|C_{G}(H)\right|=\left|K_{2}\right|\left|C_{G}\left(K_{2}\right)\right|,
$$

and consequently $\left|K_{1}: L\right|=\left|C_{G}\left(K_{2}\right): C_{G}(H)\right|=\left|K_{2}^{*}: L^{*}\right|$.

In the situation that $w \geq 3$, then $H=K_{1} K_{2}=K_{3} K_{2}$ where $K_{1} \cap K_{2}=$ $K_{2} \cap K_{3}=L$ and thus $\left|K_{i}\right|=\left|K_{j}\right|$ for all $i, j$ with $1 \leq i, j \leq w$. This additionally yields $K_{i} / L \cong K_{j} / L$ for all $i, j$. From the Isomorphism Theorems it follows that $\left|H / K_{1}\right|=\left|K_{1} / L\right|^{2}$.

The same arguments applied to the quasi-antichain $\llbracket L^{*}, H^{*} \rrbracket$ yield the remaining assertions.

Proposition 3. If $w \geq 3$ then $\left[H, H^{*}\right] \leq L \cap L^{*}=C_{G}\left(H H^{*}\right)$. Additionally, $H / L$ and $H^{*} / L^{*}$ are isomorphic elementary abelian p-groups. In particular, if $G=H$ and $G \in \mathcal{C D}(G)$ then $G / Z(G)$ and $[G, G]$ are elementary abelian p-groups.

Proof. Since $w \geq 3$, there exist at least three distinct atoms $K_{1}, K_{2}$, and $K_{3}$ in the interval $\llbracket L, H \rrbracket$ in $\mathcal{C D}(G)$. By Proposition 2 .

$$
\left[K_{1}, K_{2} K_{3}\right] \leq\left\langle\left[K_{1}, K_{3}\right]\left[K_{1}, K_{2}\right]\right\rangle \leq L .
$$

Therefore $K_{1} / L$ centralizes $K_{2} K_{3} / L=H / L$. By symmetry, the same holds for $K_{2} / L$; consequently $H=K_{1} K_{2}$ centralizes $H / L$ and $H / L$ is abelian. Similarly $H^{*}$ centralizes $H^{*} / L^{*}$ and the latter is abelian.

Since $K_{i}$ normalizes every $K_{j}$, it also normalizes every $K_{j}^{*}$. Therefore $\left[K_{i}, H^{*}\right]=$ $\left[K_{i}, K_{1}^{*} K_{2}^{*}\right] \leq K_{2}^{*}$ and $\left[K_{i}, H^{*}\right]=\left[K_{i}, K_{1}^{*} K_{3}^{*}\right] \leq K_{3}^{*}$, so that $\left[K_{i}, H^{*}\right] \leq L^{*}$. Similarly $\left[K_{2}, H^{*}\right] \leq L^{*}$ and thus $\left[H, H^{*}\right] \leq L^{*}$. In the same way, $\left[H, H^{*}\right] \leq L$. By the 
duality of the Chermak-Delgado lattice, $L \cap L^{*}=C_{G}\left(H H^{*}\right)$ so $\left[H, H^{*}\right] \leq L \cap L^{*}=$ $C_{G}\left(H H^{*}\right)$, as claimed.

Applying Proposition 10 subgroup $A_{1}$ where $A_{1} / L=\Omega_{1}(H / L)$ is a member of $\mathcal{C D}(G)$. Since $\mathcal{C D}(G)$ is a quasi-antichain, either $A=H$ or there exists $i$ such that $1 \leq i \leq w$ where $K_{i}=A$. At minimum, $K_{i} / L$ is an elementary abelian $p$-group but, as $K_{i} / L \cong K_{j} / L$ for all $i, j$, we have $H / L$ is an elementary abelian $p$ group. With similar reasoning, $H^{*} / L^{*}$ is an elementary abelian $p$-group and, since $|H / L|=\left|H^{*} / L^{*}\right|$, these quotients are isomorphic elementary abelian $p$-groups.

If $H=G$ and $\mathrm{Z}(G)=L$ then $G / \mathrm{Z}(G)$ is an elementary abelian $p$-group. Thus, for $x, y \in G$, we have $[x, y]^{p}=\left[x^{p}, y\right]=1$; therefore $[G, G]$ is elementary abelian.

Theorem 4. Let $G$ be a group such that $L, H \in \mathcal{C D}(G)$ with the interval $\llbracket L, H \rrbracket$ in $\mathcal{C D}(G)$ a quasi-antichain of width $w \geq 3$. There exists a prime $p$ and positive integers $a, b$ with $b \leq a$ such that $H / L$ and $C_{G}(L) / C_{G}(H)$ are elementary abelian p-groups of order $p^{2 a}$ and $w=p^{b}+1$.

Proof. The existence of the prime $p$ and the fact that $H / L$ and $H^{*} / L^{*}$ are elementary abelian $p$-groups were established in Proposition 3. From Proposition 2, we know $H / L=K_{1} / L \times K_{2} / L$. Let $i \geq 3$; the subgroup $K_{i} / L$ projects onto each coordinate under the natural projection maps and intersects each of $K_{1} / L$ and $K_{2} / L$ trivially. Thus $K_{i} / L$ is a subdirect product and there exists an isomorphism $\overline{\beta_{i}}: K_{1} / L \rightarrow K_{2} / L$ such that $K_{i} / L=\left\{(k L) \overline{\beta_{i}}(k L) \mid k \in K_{1}\right\}$. Choose $\beta_{i}(k) \in K_{2}$ with $\overline{\beta_{i}}(k L)=\beta_{i}(k) L$; then $K_{i} / L=\left\{k \beta_{i}(k) L \mid k \in K_{1}\right\}$. Similarly, there exists an isomorphism $\overline{\alpha_{i}}: K_{1}^{*} / L^{*} \rightarrow K_{2}^{*} / L^{*}$ where $\overline{\alpha_{i}}\left(m L^{*}\right)=\alpha_{i}(m) L^{*}$ for each $m \in K_{1}^{*}$ and $K_{i}^{*} / L^{*}=\left\{m \alpha_{i}(m) L^{*} \mid m \in K_{1}^{*}\right\}$.

For $i, j$ such that $3 \leq i, j \leq w$, let $\Delta_{i, j}=\left\{k \beta_{i}(k) \beta_{j}(k) \mid k \in K_{1}\right\}$ and $\Delta_{i, j}^{*}=$ $\left\{m \alpha_{i}(m) \alpha_{j}(m) \mid m \in K_{1}^{*}\right\}$; additionally define $K_{i, j}=\Delta_{i, j} L$ and $K_{i, j}^{*}=\Delta_{i, j}^{*} L^{*}$. Since $\left[K_{1}, K_{2}\right] \leq L$ and the functions $\overline{\beta_{i}}, \overline{\beta_{j}}$, are homomorphisms, it follows that $K_{i, j} \leq H$. Also observe that if $k \beta_{i}(k) \beta_{j}(k) L=k^{\prime} \beta_{i}\left(k^{\prime}\right) \beta_{j}\left(k^{\prime}\right) L$ then $k L=k^{\prime} L$, because $K_{1} \cap K_{2}=L$. Therefore $\left|K_{i, j} / L\right|=\left|K_{1} / L\right|$. Corresponding facts are true regarding $K_{i, j}^{*}$.

Our goal is to show that $K_{i, j}$ is one of the atoms in $\llbracket L, H \rrbracket$, so we calculate $m\left(K_{i, j}\right)$. From the definitions, clearly $\left[k_{1}, m_{1}\right]=\left[k_{2}, m_{2}\right]=1$ when $k_{i} \in K_{i}$ and $m_{i} \in K_{i}^{*}$ for $i=1,2$. By this information and the fact that $\left[H, H^{*}\right]$ is centralized by $H$ and $H^{*}$, if $k \in K_{1}$ and $m \in K_{1}^{*}$ then we obtain

$$
1=\left[k \beta_{i}(k), m \alpha_{i}(m)\right]=\left[k, \alpha_{i}(m)\right]\left[\beta_{i}(k), m\right]
$$

for all $i$ such that $3 \leq i \leq w$. Given $k \in K_{1}$ and $m \in K_{1}^{*}$, if $3 \leq i, j \leq w$ then

$$
\begin{aligned}
& {\left[k \beta_{i}(k) \beta_{j}(k), m \alpha_{i}(m) \alpha_{j}(m)\right]} \\
& \quad=\left[k, \alpha_{j}(m)\right]\left[\beta_{i}(k), \alpha_{j}(m)\right]\left[\beta_{j}(k), \alpha_{i}(m)\right]\left[\beta_{j}(k), m\right] \\
& \quad=\left[k, \alpha_{j}(m)\right]\left[\beta_{j}(k), m\right] \\
& \quad=1 .
\end{aligned}
$$

By $\left[H, L^{*}\right]=\left[H^{*}, L\right]=1$ and the above calculation, $K_{i, j}^{*} \leq C_{G}\left(K_{i, j}\right)$. Because $\left|K_{i, j}\right|=\left|K_{1}\right|$ and $\left|K_{i, j}^{*}\right|=\left|K_{1}^{*}\right|$, therefore $m\left(K_{i, j}\right)=m\left(K_{1}\right)$ and $K_{i, j} \in \mathcal{C D}(G)$. Thus for each $i, j$ with $3 \leq i, j \leq w$, either $K_{i, j}=K_{h}$ for some $h$ such that $3 \leq h \leq w$ or $K_{i, j}=K_{1}$. Setting $\beta_{2}(k)=1$ for all $k \in K_{1}$, it follows that $\left\{k \beta_{i}(k) \beta_{j}(k) L \mid k \in K_{1}\right\}=\left\{k \beta_{h}(k) L \mid k \in K_{1}\right\}$ for some $h$ with $2 \leq h \leq w$. Notice that if $k \beta_{i}(k) \beta_{j}(k) L=k^{\prime} \beta_{h}\left(k^{\prime}\right) L$ then $k L=k^{\prime} L$ and $\beta_{i}(k) \beta_{j}(k) L=\beta_{h}(k) L$, because $K_{1} \cap K_{2}=L$. 
Fix a $k_{1} \in K_{1} \backslash L$. Let $\Lambda=\left\{\beta_{2}\left(k_{1}\right), \beta_{3}\left(k_{1}\right), \ldots \beta_{w}\left(k_{1}\right)\right\}$ and $R=\Lambda \cdot L$. By what we have shown in the preceding paragraphs, $R \leq H$ and, as $K_{i} \cap K_{j}=L$ for $i \neq j$, the set $\Lambda$ is a transversal for $L$ in $R$. Hence $|R / L|=|\Lambda|=w-1$. Since $R \leq K_{2}$, it follows that $w-1$ divides $p^{a}$.

\section{Quasi-antichain Chermak-Delgado Lattices}

We study here the groups $G$ such that $\mathcal{C D}(G)$ is a quasi-antichain and $G \in$ $\mathcal{C D}(G)$, meaning that $G=H=H^{*}$ and $\mathrm{Z}(G)=L=L^{*}$ in the notation of the first section. Additionally, the subgroups $K_{i}^{*}$ are now atoms of $\llbracket L, H \rrbracket$; notice $K_{i}^{*}=K_{i}$ if and only if $K_{i}$ is abelian.

When studying groups of this type, the added condition that $[G, G]$ be cyclic imposes very strong restrictions on the structure of the group, as seen in the next proposition.

Proposition 5. Let $G \in \mathcal{C D}(G)$ and $[G, G]$ be cyclic. Then $\mathcal{C D}(G)$ is a quasiantichain of width $w \geq 3$ with $G \in \mathcal{C D}(G)$ if and only if there exists a prime $p$ such that $|[G, G]|=p$ and $G / Z(G) \cong C_{p} \times C_{p}$. In this case $w=p+1$.

Proof. Let $[G, G]$ is cyclic and $G \in \mathcal{C D}(G)$. Suppose first that $\mathcal{C D}(G)$ is a quasiantichain of width $w \geq 3$. We know that there exists a prime $p$ such that $G / \mathrm{Z}(G)$ and $[G, G]$ are elementary abelian $p$-groups by Proposition 3 , Therefore $[G, G]$ has order $p$ but, more importantly, all $U \leq G$ such that $\mathrm{Z}(G) \leq U$ are centralizers by [7, Satz]. In particular, a maximal subgroup $M<G$ is a centralizer so there exists $U>\mathrm{Z}(P)$ with $M=C_{G}(U)$ and $m(M)=\frac{|G|}{p}\left|C_{G}(X)\right| \geq|G||\mathrm{Z}(G)|$. Yet $G \in \mathcal{C D}(G)$, so $M, U \in \mathcal{C D}(G)$. The Chermak-Delgado lattice of $G$ is a quasiantichain of width at least 3 so by Proposition 2, $|M|=|U|$ and thus $|G / \mathrm{Z}(P)|=p^{2}$.

Now suppose there exists a prime $p$ such that $|[G, G]|=p$ and $G / \mathrm{Z}(G) \cong C_{p} \times C_{p}$. In this case, all $p+1$ subgroups $U$ such that $\mathrm{Z}(G)<U<G$ are abelian, have order $p|\mathrm{Z}(G)|$, and have measure $p^{2}|\mathrm{Z}(G)|^{2}$, which also equals the measure of $G$. Therefore $\mathcal{C D}(G)=\{U \leq G \mid \mathrm{Z}(G) \leq U\}$ is a quasi-antichain of width $p+1$ with $G \in \mathcal{C D}(G)$.

The next theorem justifies our attention on $p$-groups while studying groups with a quasi-antichain Chermak-Delgado lattice. The proof of Theorem 6 requires the observation: Let $M$ and $N$ be any pair of finite groups. The modularity of the Chermak-Delgado lattice implies that every maximal chain in the lattice has the same length. For example, all maximal chains in $\mathcal{C D}(G)$ have length 2 because $\mathcal{C D}(G)$ is a quasi-antichain. That $\mathcal{C D}(M \times N) \cong \mathcal{C D}(M) \times \mathcal{C D}(N)$ as lattices [3] gives that the length of a maximal chain in $M \times N$ is the sum of the lengths of maximal chains in $M$ and $N$.

Theorem 6. If $G$ is a group with $\mathcal{C D}(G)$ a quasi-antichain of width $w \geq 3$ and $G \in$ $\mathcal{C D}(G)$ then $G$ is nilpotent of class 2; in fact, there exists a prime $p$, a nonabelian Sylow p-subgroup $P$ with nilpotence class 2 , and an abelian Hall $p^{\prime}$-subgroup $Q$ such that $G=P \times Q, P \in \mathcal{C D}(P)$, and $\mathcal{C D}(G) \cong \mathcal{C D}(P)$ as lattices. Moreover there exist positive integers $a, b$ with $b \leq a$ such that $|G / Z(G)|=|P / Z(P)|=p^{2 a}$ and $w=p^{b}+1$.

Proof. Note that $G$ is nilpotent, by Proposition 3, but nonabelian and the length of a maximal chain in $\mathcal{C D}(G)$ is 2 . If $G=Q_{1} \times Q_{2}$ where $Q_{1}$ and $Q_{2}$ are Hall $\pi-, \pi^{\prime}$ subgroups of $G$, respectively, then $\mathcal{C D}(G) \cong \mathcal{C D}\left(Q_{1} \times Q_{2}\right)$; as a consequence of the 
additivity of chain length over a direct product, if both $Q_{1}$ and $Q_{2}$ are nonabelian then $\mathcal{C D}\left(Q_{i}\right)=\left\{Q_{i}, \mathrm{Z}\left(Q_{i}\right)\right\}$ for $i=1,2$. However, this implies $\mathcal{C} \mathcal{D}\left(Q_{1} \times Q_{2}\right)$ is a quasi-antichain of width 2. Consequently, exactly one of $Q_{1}$ or $Q_{2}$ is abelian and the Chermak-Delgado lattice of the nonabelian factor is isomorphic (as lattices) to $\mathcal{C D}(G)$. Therefore there exists a unique prime $p$ such that $G=P \times Q$ where $P$ is a nonabelian Sylow $p$-subgroup of $G$ and $Q$ is an abelian Hall $p^{\prime}$-subgroup of $G$, with $\mathcal{C D}(G) \cong \mathcal{C D}(P)$ as lattices. The rest follows from Proposition 2 and Theorem 4 .

We investigated the number of abelian atoms that is permitted in a quasiantichain Chermak-Delgado lattice. The final theorem of this section records our contributions in this direction.

Theorem 7. Let $G$ be a p-group with $\mathcal{C D}(G)$ a quasi-antichain of width $w \geq 3$ and suppose $|G / Z(G)|=p^{2 a}$ for a positive integer a. Let $t$ be the number of abelian atoms in $\mathcal{C D}(G)$ and $u$ be the number of pairs of nonabelian atoms.

(1) If $t=0$ then $p$ is odd.

(2) If $t=1$ then $p=2$.

(3) If $t \geq 2$ then there exists a positive integer $c \leq a$ such that $t=p^{c}+1$. In particular, $p-1$ divides $t-2$; if $p$ is odd then $p^{c}$ divides $u$ and if $p=2$ then $t \geq 3$ and $2^{c-1}$ divides $u$.

(4) If $t \geq 2$ and $u \geq 1$ then $3 \leq t \leq 2 u+1$ when $p=2$ and $2 \leq t \leq u+1$ when $p$ is odd.

(5) If $t \geq 3$ then $t \geq p+1$.

Proof. Theorem 4 tells us that $w=p^{b}+1$ for some positive integer $b \leq a$, but also $w=t+2 u$ as set up by the notation. If $t=0$ then $w=2 u=p^{b}+1$, necessarily forcing $p$ to be odd. If $t=1$ then $2 u=p^{b}$; clearly $p$ must equal 2 in this case.

Suppose that $t \geq 2$; we continue here with the same notation and set up as in the proof of Theorem 4 except we add the condition that $K_{1}$ and $K_{2}$ are abelian atoms. Recall the fixed $k_{1} \in K_{1} \backslash L$ and that $\beta_{2}(k)=1$ for all $k \in K_{1}$. Set $\Gamma=\left\{\beta_{i}\left(k_{1}\right) \mid 2 \leq i \leq w\right.$ and $\left.K_{i}=K_{i}^{*}\right\}$, a subset of $\Lambda$.

Let $i, j \geq 3$ and assume that $K_{i}$ and $K_{j}$ are abelian atoms; we show that $K_{i, j}$ is also abelian. We use the functions $\alpha_{i}$ defined in the proof of Theorem 4 . Observe that $\alpha_{i}(k)$ now differs from $\beta_{i}(k)$ only by an element in $\mathrm{Z}(G)$, for all $k \in K_{1}$. The calculation below follows:

$$
\left[k \beta_{i}(k) \beta_{j}(k), k^{\prime} \beta_{i}\left(k^{\prime}\right) \beta_{j}\left(k^{\prime}\right)\right]=\left[k \beta_{i}(k) \beta_{j}(k), k^{\prime} \alpha_{i}\left(k^{\prime}\right) \alpha_{j}\left(k^{\prime}\right)\right]=1
$$

for all $k, k^{\prime} \in K_{1}$. Therefore $K_{i, j}$ is also an abelian atom in $\mathcal{C D}(G)$. Since $K_{i, j} \neq K_{2}$, it follows that $\left.\beta_{(} k_{1}\right) \beta_{j}\left(k_{1}\right) \mathrm{Z}(G)=\beta_{h}\left(k_{1}\right) \mathrm{Z}(G)$ for some $\beta_{h}\left(k_{1}\right) \in \Gamma$. Consequently $\Gamma \mathrm{Z}(G) \leq K_{2}$ and since $\Gamma \subseteq \Lambda$, the elements of $\Gamma$ are distinct. Therefore $|\Gamma \mathrm{Z}(G) / \mathrm{Z}(G)|=|\Gamma|=t-1$ divides $p^{a}$ and there exists a positive integer $c$ such that $t-1=p^{c}$.

Since $t=p^{c}+1$, clearly if $p=2$ and $t \geq 2$ then $t=2^{c}+1 \geq 3$ but, for all primes $p$, it is true that $p-1$ divides $p^{c}-1=t-2$. Observe, for part (5), that if $t \geq 3$ then $t \geq p+1$ is necessary for $t-2$ to be a multiple of $p-1$. To complete the proof of part (3), notice that $2 u=w-t=p^{c}\left(p^{b-c}-1\right)$, so that $u=\frac{1}{2} p^{c}\left(p^{b-c}-1\right)$. If $p=2$ then $2^{c-1}$ divides $u$, and if $p$ is odd then $u$ must be divisible by $p^{c}$. This completes the assertions in part (3). 
Continuing with part (4), suppose that $t \geq 2$ and $u \geq 1$. Then $b>c$, so $p^{b-c}-1>p-1$. If $p$ is odd, this implies $\frac{1}{2}\left(p^{b-c}-1\right) \geq 1$ and consequently

$$
t=p^{c}+1 \leq p^{c}\left(\frac{p^{b-c}-1}{2}\right)+1=u+1 .
$$

If $p=2$ then $2^{b-c}-1 \geq 1$, so that

$$
t=2^{c}+1 \leq 2^{c}\left(2^{b-c}=1\right)+1=2 u+1 .
$$

Thus when $t \geq 2$ and $u \geq 1$, the inequalities asserted in part (4) of the theorem are true.

Corollary 8. While there exist finite groups with Chermak-Delgado lattice a quasiantichain of width 6 , there does not exist such a group with exactly 4 abelian atoms in its Chermak-Delgado lattice.

Proof. An extraspecial group of order $5^{3}$ has a Chermak-Delgado lattice that is a quasi-antichain of width 6 by Proposition 5 . If we assume that $G$ is a finite group with $\mathcal{C D}(G)$ a quasi-antichain of width 6 having exactly 4 abelian atoms then we know that $G$ has a Sylow 5 -subgroup $P$ with $\mathcal{C D}(G) \cong \mathcal{C D}(P)$ as lattices by Proposition 6 and Theorem [4. Theorem 7 forces $4=t \leq u+1=2$; thus $G$ cannot exist.

\section{EXAMPLES}

In this section we construct several examples of $p$-groups having a quasi-antichain for their Chermak-Delgado lattice. The first two examples show that every possible quasi-antichain of width 2 can be realized as the Chermak-Delgado lattice of a $p$ group.

(1) A group $G$ where $\mathcal{C D}(G)$ is a quasi-antichain of width 2 with no abelian atoms: Let $H$ be any group with $\mathcal{C D}(H)=\{H, \mathrm{Z}(H)\}$. A family of $p$-groups, each member of which having such a Chermak-Delgado lattice, was described in [2].

Define $G=H \times H$. In [3] it was established that $\mathcal{C D}(G)$ is a quasiantichain of width 2 with atoms $\mathrm{Z}(H) \times H$ and $H \times \mathrm{Z}(H)$. Clearly $H \times$ $\mathrm{Z}(H)=C_{G}(\mathrm{Z}(H) \times H)$.

(2) A group $P$ such that $\mathcal{C D}(P)$ is a quasi-antichain of width 2 with both atoms abelian: Let $P=\left\langle m_{1}, m_{2}, n_{1}, n_{2}\right\rangle$ where each element has order $p$ and

$$
\left[m_{1}, m_{2}\right]=\left[n_{1}, n_{2}\right]=1, \quad\left[m_{i}, n_{j}\right]=z_{i j} \in \mathrm{Z}(P) \text { for } i, j \in\{1,2\},
$$

and $\mathrm{Z}(P)=\left\langle z_{i, j} \mid i, j \in\{1,2\}\right\rangle$ is elementary abelian of order $p^{4}$. Clearly $P$ is nilpotent of class 2 with order $p^{8}$ and Chermak-Delgado measure $p^{12}$. Let $M=\left\langle m_{1}, m_{2}\right\rangle \mathrm{Z}(P)$ and $N=\left\langle n_{1}, n_{2}\right\rangle \mathrm{Z}(P)$. It's a straightforward calculation to show that $C_{P}(m)=M$ whenever $m \in M \backslash \mathrm{Z}(P)$ and $C_{P}(n)=$ $N$ whenever $n \in N \backslash \mathrm{Z}(P)$, whereas $C_{P}(x)=\langle x\rangle \mathrm{Z}(P)$ for all $x \in P \backslash(M \cup N)$. Thus of all subgroups containing $\mathrm{Z}(P)$, only $M$ and $N$ have the largest measure, which is $p^{12}$. Since $m_{P}(M)=m_{P}(N)=m_{P}(\mathrm{Z}(P))=p^{12}$ and no other subgroups have this measure, $\mathcal{C D}(P)$ is a quasi-antichain of width 2 (containing $P$ ) such that both atoms are abelian. 
We now show that for every prime $p$ and every positive integer $n$, there exists a $p$-group whose Chermak-Delgado lattice is a quasi-antichain of width $p^{n}+1$ with all atoms abelian.

Proposition 9. Let $p$ be a prime and $n$ a positive integer. Let $P$ be the group of all $3 \times 3$ lower triangular matrices over $\mathbf{G F}\left(p^{n}\right)$ with 1 s along the diagonal. The Chermak-Delgado lattice of $P$ is a quasi-antichain of width $p^{n}+1$ and all subgroups in the middle antichain are abelian.

Proof. By Exercise 39 in [5, III.16], $P$ has exactly $p^{n}+1$ abelian subgroups of maximal order equal to $p^{2 n}$; these subgroups have measure $p^{4 n}=m(P)$. If $x \in$ $P \backslash \mathrm{Z}(P)$, it is easy to check that $\left|C_{P}(x)\right|=p^{2 n}$. Therefore if $U \in \mathcal{C D}(P)$ with $\mathrm{Z}(P)<U<P$ then $\left|C_{P}(U)\right| \leq p^{2 n}$ and $|U|=\left|C_{P}\left(C_{P}(U)\right)\right| \leq p^{2 n}$. If follows that $m^{*}(P)=p^{4 n}$ and $|U|=\left|C_{P}(U)\right|=p^{2 n}$. If $U \neq C_{P}(U)$ then $U \cap C_{P}(U)=\mathrm{Z}(P)$ since $U \cap C_{P}(U) \in \mathcal{C D}(P)$. But then for $x \in U \backslash \mathrm{Z}(P)$ we have $C_{P}(x)=C_{P}(U)$ by order considerations and therefore $x \in U \cap C_{P}(U)=\mathrm{Z}(P)$, a contradiction. Thus $U=C_{P}(U)$ is one of the abelian subgroups of maximal order and the assertion follows.

Extraspecial groups of order $p^{3}$ are examples where each of the $p+1$ atoms in the quasi-antichain is abelian (Proposition 5); the next two propositions construct $p$-groups where the Chermak-Delgado lattice is a quasi-antichain of width $p+1$ and, depending on the value of $p$ modulo 4 , the number of abelian atoms is either 0,1 , or 2 .

Proposition 10. Given any prime $p$ there exists a group $P$ of order $p^{9}$ such that $\mathcal{C D}(P)$ is a quasi-antichain of width $p+1$. In this example: if $p=2$ then exactly one of the three atoms of $\mathcal{C D}(P)$ is abelian, when $p \equiv 1$ modulo 4 then exactly two of the $p+1$ atoms of $\mathcal{C D}(P)$ are abelian, and if $p \equiv 3$ modulo 4 then none of the atoms in $\mathcal{C D}(P)$ are abelian.

Proof. Let $P$ be generated by $\left\{x_{1}, x_{2}, x_{3}, y_{1}, y_{2}, y_{3}\right\}$ with defining relationships $x_{i}^{p}=$ $y_{i}^{p}=1$ and $\left[x_{i}, y_{j}\right]=1$ for all $i, j$ such that $1 \leq i, j \leq 3, \mathrm{Z}(P)=\left\langle z_{1,2}, z_{1,3}, z_{2,3}\right\rangle$ is elementary abelian with order $p^{3}$, and $\left[x_{i}, x_{j}\right]=\left[y_{i}, y_{j}\right]=z_{i j}$ for every $i, j$ with $1 \leq$ $i<j \leq 3$. Let $M_{0}=\left\langle x_{1}, x_{2}, x_{3}\right\rangle \mathrm{Z}(P)$ and $M_{p}=\left\langle y_{1}, y_{2}, y_{3}\right\rangle \mathrm{Z}(P)$. For $1 \leq i \leq p-1$, let $M_{i}=\left\langle x_{1} y_{1}^{i}, x_{2} y_{2}^{i}, x_{3} y_{3}^{i}\right\rangle \mathrm{Z}(P)$. We show that $\mathcal{C D}(P)=\left\{P, \mathrm{Z}(P), M_{i} \mid 0 \leq i \leq p\right\}$.

Observe that $P$ is the central product of $M_{0}$ with $M_{p}$ and $\mathrm{Z}(P)=M_{0} \cap M_{p}$. Additionally $C_{P}\left(M_{0}\right)=M_{p}$ and vice versa, yielding $m_{P}(P)=m_{P}\left(M_{0}\right)=m_{P}\left(M_{p}\right)=$ $p^{12}$. It is easy to show that if $x \in M_{0} \backslash \mathrm{Z}(P)$ then $C_{M_{0}}(x)=\langle x\rangle \mathrm{Z}(P)$ and $C_{P}(x)=\langle x\rangle M_{p}$. It follows that $C_{P}(\langle x, y\rangle)=\langle x, y\rangle \mathrm{Z}(P)$ whenever $x \in M_{0} \backslash \mathrm{Z}(P)$ and $y \in M_{p} \backslash \mathrm{Z}(P)$.

Let there exist $a_{i}, b_{i} \in \mathbb{Z} / p \mathbb{Z}$ such that $x=x_{1}^{a_{1}} x_{2}^{a_{2}} x_{3}^{a_{3}} \in M_{0} \backslash \mathrm{Z}(P)$ and $y=$ $y_{1}^{b_{1}} y_{2}^{b_{2}} y_{3}^{b_{3}} \in M_{p} \backslash \mathrm{Z}(P)$. Assume that at least one of $a_{1}, a_{2}, a_{3}$ and one of $b_{1}, b_{2}, b_{3}$ are non-zero. For $x^{\prime} y^{\prime}$ with similar structure, $x^{\prime} y^{\prime} \in C_{P}(x y)$ if and only if $\left[x, x^{\prime}\right]=$ $\left[y^{\prime}, y\right]$. Further decomposing the commutators reveals

$$
\left[x, x^{\prime}\right]=\prod_{1 \leq i<j \leq 3} z_{i j}^{a_{i} a_{j}^{\prime}-a_{j} a_{i}^{\prime}} \text { and }\left[y^{\prime}, y\right]=\prod_{1 \leq i<j \leq 3} z_{i j}^{b_{i}^{\prime} b_{j}-b_{j}^{\prime} b_{i}} ;
$$

Thus $\left[x y, x^{\prime} y^{\prime}\right]=1$ if and only if each of the three equations $a_{i} a_{j}^{\prime}-a_{j} a_{i}^{\prime}-b_{i}^{\prime} b_{j}+$ $b_{j}^{\prime} b_{i}=0$ hold where $1 \leq i<j \leq 3$. If $\left(a_{1}, a_{2}, a_{3}\right)$ and $\left(b_{1}, b_{2}, b_{3}\right)$ are not scalar multiples then $\left\langle x, y, m_{1}^{b_{1}} m_{2}^{b_{2}} m_{3}^{b_{2}} n_{1}^{a_{1}} n_{2}^{a_{2}} n_{3}^{a_{3}}\right\rangle \mathrm{Z}(P)=C_{P}(x y)$. On the other hand, if 
there exists $k$ such that $\left(b_{1}, b_{2}, b_{3}\right)=k\left(a_{1}, a_{2}, a_{3}\right)$ then $C_{P}(x y)=\left\langle m, m_{i} n_{i}^{-k^{-1}}\right.$ $1 \leq i \leq 3\rangle \mathrm{Z}(P)=\langle m\rangle M_{-k^{-1}}$. Therefore $C_{P}\left(M_{k}\right)=M_{-k^{-1}}$ for $1 \leq k \leq p-1$ and $m_{P}\left(M_{k}\right)=p^{12}$.

It follows then that $m(U)<m\left(M_{k}\right)$ whenever $\mathrm{Z}(P)<U<M_{k}$. Additionally, if $U \leq P$ and there exist $u_{1}, u_{2} \in U$ where $u_{1} \in M_{k}$ and $u_{2} \in M_{k^{\prime}}$ with $k \neq k^{\prime}$ then $C_{P}(U) \leq \mathrm{Z}(P)$. Hence $m^{*}(P)=p^{12}$ and $\mathcal{C D}(P)=\left\{P, \mathrm{Z}(P), M_{k} \mid 0 \leq k \leq p\right\}$.

Since $C_{P}\left(M_{k}\right)=M_{-k^{-1}}$ for $1 \leq k \leq p-1$, there exists an abelian atom of $\mathcal{C D}(P)$ if and only if $p=2$ or $p \equiv 1$ modulo 4 . When $p=2$ then $M_{1}$ is the unique abelian atom and if $p \equiv 1$ modulo 4 then $M_{1}$ and $M_{p-1}$ are both abelian, but no other atom in $\mathcal{C D}(P)$ is abelian. When $p \equiv 3$ modulo 4 then there do not exist any abelian atoms in $\mathcal{C D}(P)$.

Proposition 11. Let $p$ be a prime. There exists a group $Y$ of order $p^{9}$ such that $\mathcal{C D}(Y)$ is a quasi-antichain of width $p+1$. In this example, if $p=2$ then exactly one of the three atoms of $\mathcal{C D}(Y)$ is abelian and if $p$ is odd then exactly two of the $p+1$ atoms are abelian.

Proof. Define $P$ as in Proposition[10 except designate that $\left[n_{i}, n_{j}\right]=z_{i j}^{-1}$. The same arguments made earlier will now show that $C_{P}\left(M_{i}\right)=M_{i^{-1}}$ for $i=1,2, \ldots, p-1$. This forces $M_{1}$ and $M_{p-1}$ to be abelian, yet the remaining facts still stand.

In lattice theory [1, Chapter 1, Section 2], a lattice $\mathcal{L}$ has a duality $\theta: \mathcal{L} \rightarrow \mathcal{L}$ if $\theta$ is a bijection and if $A, B \in \mathcal{L}$ with $A \leq B$ implies $\theta(B) \leq \theta(A)$. Such a duality need not have order 2 as a function; in the case of the Chermak-Delgado lattice the duality does have order 2. In particular, the examples in this section show that all possible types of quasi-antichains of width 4 with duality of order 2 occur as Chermak-Delgado lattices of 3-groups and those of width 3 with duality of order 2 occur as Chermak-Delgado lattices of 2-groups.

This leaves several questions open for investigation, including: Which values of $t$ (in the notation of Theorem 7) are possible in quasi-antichain Chermak-Delgado lattices of width $w=p^{n}+1$ when $n>1$ ? That is, which dualities can be realized by the centralizer map? The first open case is when $w=5$ and $t=3$. And, are there examples of groups $G$ with $G \in \mathcal{C D}(G)$ and $\mathcal{C D}(G)$ a quasiantichain where $t=0$ and either $p=2$ or $p \equiv 1$ modulo 4 ?

\section{ACKNOWLEDGEMENTS}

The research of the second author was supported by Projecto MTM2010-19938C03-02, Ministerio de Ciencia e Innovación, Spain. The research of the third author was supported by a Scholarly and Creative Activity Grant from Oswego State University.

\section{REFERENCES}

1. G. Birkhoff, Lattice Theory, American Mathematical Society, 1967.

2. B. Brewster, P. Hauck, and E. Wilcox, Groups whose Chermak-Delgado Lattice is a Chain, J. Group Th. 17 (2014), $253-279$.

3. B. Brewster and E. Wilcox, Some Groups with Computable Chermak-Delgado Lattices, Bull. Aus. Math. Soc. 86 (2012), $29-40$.

4. A. Chermak and A. Delgado, A measuring argument for finite groups, Proc. AMS 107 (1989), no. $4,907-914$.

5. B. Huppert, Endliche Gruppen I, Springer-Verlag, 1967.

6. I. Martin Isaacs, Finite Group Theory, American Mathematical Society, 2008. 
7. M. Reuther, Endliche Gruppen, in denen alle das Zentrum enthaltenden Untergruppen Zentralisatoren sind, Arch. Math. 29 (1977), 45 - 54.

Ben Brewster, Department of Mathematical Sciences, Binghamton University, BingHAMTON, NEW YORK, 13905

E-mail address: ben@math.binghamton.edu

Peter Hauck, Fachbereich Informatik, Eberhard-Karls-Universität Tübingen, Tübingen, Germany

E-mail address: hauck@informatik.uni-tuebingen.edu

Elizabeth Wilcox, Mathematics Department, Oswego State University, Oswego, New YORK, 13126

E-mail address: elizabeth.wilcox@oswego.edu 